\title{
Introduction to special topic - Resting-state brain activity: implications for systems neuroscience
}

\author{
Lucina Q. Uddin ${ }^{1,2 *}$ and Vinod Menon ${ }^{1,2,3 *}$ \\ Department of Psychiatry and Behavioral Sciences, Stanford University School of Medicine, Stanford, CA, USA \\ 2 Program in Neuroscience, Stanford University School of Medicine, Stanford, CA, USA \\ ${ }^{3}$ Department of Neurology and Neurological Sciences, Stanford University School of Medicine, Stanford, CA, USA \\ Correspondence: *lucina@stanford.edu; **menon@stanford.edu
}

Research on resting-state brain activity using fMRI offers a novel approach for understanding brain organization at the systems level. Resting-state fMRI (rsfMRI) examines spatial synchronization of intrinsic fluctuations in blood oxygenation level dependent (BOLD) signals arising from neuronal and synaptic activity that is present in the absence of overt cognitive information processing. Since the discovery of coherent spontaneous fluctuations within the somatomotor system (Biswal et al., 1995), a growing number of studies have shown that many of the brain areas engaged during various cognitive tasks also form coherent large-scale brain networks that can be readily identified using rsfMRI (Smith et al., 2009). These studies are beginning to provide new insights into the functional architecture of the human brain. This special topic synthesizes current knowledge about resting-state brain activity and discusses implications for understanding brain function and dysfunction from a systems neuroscience perspective. Reviews written by experts in the field provide perspectives on important conceptual, methodological, and empirical questions that need to be addressed in the next years. Additionally, this collection includes original research articles addressing questions related to the nature, origins, and functions of resting-state brain activity.

The low cognitive demand and relatively short duration of rsfMRI scans make them well suited for studying pediatric and clinical populations with low tolerance for the MRI scanner environment. The review by Uddin et al. (2010) summarizes rsfMRI studies to date in children and adolescents, and describes new insights that have emerged about the typical and atypical development of functional brain networks, a topic also examined in an empirical study by Littow et al. (2010). The review by Fox and Greicius (2010) highlights advantages of examining the restingstate signal for clinical applications and discusses methodological issues that need to be resolved to facilitate translational applications of rsfMRI. A number of important clinical applications are already emerging as emphasized by the studies of functional connectivity in premature children (Damaraju et al., 2010), adolescents with schizotypal traits (Lagioia et al., 2010), major depression (Horn et al., 2010), and aging (Langan et al., 2010). Furthermore, rsfMRI studies in healthy individuals are continuing to provide new insights into cortical and subcortical functional networks and their interconnections with a high degree of specificity, as demonstrated by Barnes et al. (2010) in the basal ganglia and Fair et al. (2010) in the thalamus.

As the neuroimaging field begins to incorporate rsfMRI into its arsenal of tools, increasingly sophisticated methods are being developed to maximize its potential contribution to systems neuroscience. Cole et al. (2010) provide a timely review of current methods and describe their strengths and limitations with respect to analysis and interpretation of rsfMRI data. A number of papers describe new tools and methods that are being developed in the field, as described in the studies by Chao-Gan and Yu-Feng (2010) (Benjaminsson et al., 2010; Liu et al., 2010). Graph theoretical analyses offer insights into brain networks at a global level, as discussed by Fornito et al. (2010) and reviewed by Wang et al. (2010). These methodological and technical advances have paved the way for increasingly sophisticated insights into the topology of human brain networks.

RsfMRI studies would, of course, not be meaningful if they did not have an underlying neurophysiological correlate. The review by Jerbi et al. (2010) emphasizes the links between rsfMRI connectivity and inter-areal synchronization observed with intracranial EEG, and they describe how intracranial EEG studies can provide insights into transient neural processes underlying task-induced deactivation. Maier et al. (2010) take this a step further and describe original research on the laminar pattern of spontaneous activity in primate visual cortex. Their demonstration that functional compartmentalization in superficial and deep layers found during rest, was also preserved when a neuron's receptive field was stimulated during a visual task, suggests that even at this level of brain organization, resting-state activity imposes massive constraints on stimulus processing. Sadaghiani et al. (2010) provide both a theoretical and an experimental perspective on how intrinsic brain activity influences task-evoked activity and perceptual response variability. Clearly, more work is needed to better understand the relationship between resting-state and task-evoked activity. This set of articles suggests that both theoretical and neurophysiological approaches have much to offer in this regard.

The reviews and empirical articles presented in this special topic reveal a complex and rapidly unfolding profile of how the human and primate brain are intrinsically organized. Advances in the field, both methodological and conceptual, will have profound implications for understanding human brain function from a systems neuroscience perspective. 


\section{REFERENCES}

Barnes, K. A., Cohen, A. L., Power, J. D., Nelson, S. M., Dosenbach, Y. B. L., Miezin, F. M., Petersen, S. E., and Sehlaggar, B. L. (2010). Identifying basal ganglia divisions in individuals using resting state functional connectivity MRI. Front. Syst. Neurosci. 4:18. doi:10.3389/fnsys.2010.00018

Benjaminsson, S., Fransson, P., and Lansner,A. (2010). A novel model-free data analysis technique based on clustering in a mutual information space: application to resting-state fMRI. Front. Syst. Neurosci. 4:34. doi:10.3389/ fnsys.2010.00034.

Biswal, B., Yetkin, F. Z., Haughton, V. M., and Hyde, J.S. (1995). Functional connectivity in the motor cortex of resting human brain using echo-planar MRI. Magn. Reson. Med. 34, 537-541.

Chao-Gan, Y., and Yu-Feng, Z. (2010). DPARSF: a MATLAB toolbox for "pipeline" data analysis of restingstate fMRI. Front. Syst. Neurosci. 4:13 doi:10.3389/fnsys.2010.00013.

Cole, D. M., Smith, S. M., and Beckmann, C.F. (2010).Advances and pitfalls in the analysis and interpretation of restingstate FMRI data. Front. Syst. Neurosci. 4:8. doi:10.3389/fnsys.2010.00008.

Damaraju, E., Phillips, J., Lowe, J. R., Ohls, R., Calhoun, V. D., and Caprihan, A. (2010). Resting-state functional connectivity differences in premature children. Front. Syst. Neurosci. 4:23 doi:10.3389/fnsys.2010.00023.

Fair, D. A., Bathula, D., Mills, K. L., Costa Dias, T. G., Blythe, M. S., Zhang, D.
Snyder, A. Z., Raichle, M. E., Stevens, A. A., Nigg, J. T., and Nagel, B. J. (2010). Maturing thalamocortical functional connectivity across development. Front. Syst. Neurosci. 4:10. doi:10.3389/fnsys.2010.00010.

Fornito,A.,Zalesky, A., and Bullmore,E. T. (2010). Networkscaling effectsingraph analytic studies of human resting-state fMRI data. Front. Syst. Neurosci. 4:22 doi:10.3389/fnsys.2010.00022.

Fox, M. D., and Greicius, M. (2010) Clinical applications of resting state functional connectivity with functional magnetic resonance imaging (fMRI). Front. Syst. Neurosci. 4:19. doi:10.3389/fnsys.2010.00019.

Horn, D. I., Yu, C., Steiner, J., Buchmann, J., Kaufmann, J., Osoba, A., Eckert, U., Zierhut, K., Schiltz, K., He, H., Biswal, B., Bogerts, B., and Walter, M. (2010). Glutamatergic and resting state functional connectivity correlates of severity in major depression - the role of pregenual anterior cingulate cortex and anterior insula. Front. Syst. Neurosci. 4:33. doi:10.3389/ fnsys.2010.00033.

Jerbi, K., Vidal, J. R., Ossandon, T., Dalal, S. S., Jung, J., Hoffmann, D., Minotti, L., Bertrand, O., Kahane, P., and Lachaux, J.-P. (2010). Exploring the electrophysiological correlates of the default-mode network with intracerebral EEG. Front. Syst. Neurosci. 4:27. doi:10.3389/fnsys.2010.00027.

Lagioia, A., Van De Ville, D., Debbané, M., Lazeyras, F., and Eliez, S. (2010). Adolescent resting state networks and their associations to schizotypal trait expression. Front. Syst. Neurosci. 4:35 doi: 10.3389/fnsys.2010.00035.

Langan, J., Peltier, S., Bo, J., Fling, B. W. Welsh, R. C., and Seidler, R. D. (2010) Functional implications of age differences in motor system connectivity. Front. Syst. Neurosci. 4:17.doi:10.3389/ fnsys.2010.00017.

Littow, H., Elseoud, A. A., Haapea, M. Isohanni, M., Moilanen, I., Mankinen, K., Nikkinen, J., Rahko, J., Rantala, H., Remes, J., Starck, T., Tervonen, O., Veijola, J., Beckmann, C., and Kiviniemi, V. J. (2010). Age-related differences in functional nodes of the brain cortex - a high model order group ICA study. Front. Syst. Neurosci. 4:32. doi:10.3389/fnsys.2010.00032.

Liu, D., Chao-Gan, Y., Ren, J., Yao, L., Kiviniemi, V. J., and Yu-Feng, Z (2010). Using coherence to measure regional homogeneity of resting-state fMRI signal. Front. Syst. Neurosci. 4:24 doi:10.3389/fnsys.2010.00024.

Maier, A., Adams, G. K., Aura, C., and Leopold, D. A. (2010). Distinct laminar domains of activity in the visual cortex during rest and stimulation. Front. Syst. Neurosci. 4:31.doi:10.3389/ fnsys.2010.00031.

Sadaghiani, S., Hesselmann, G., Friston, K. J., and Kleinschmidt, A. (2010) The relation of ongoing brain activity, evoked neural responses, and cognition. Front. Syst. Neurosci. 4:20. doi:10.3389/fnsys.2010.00020.

Smith, S. M., Fox, P. T., Miller, K. L. Glahn, D. C., Fox, P. M., Mackay, C.
E., Filippini, N., Watkins, K. E., Toro, R., Laird, A. R., and Beckmann, C. F. (2009). Correspondence of the brain's functional architecture during activation and rest. Proc. Natl. Acad. Sci. U.S.A. 106, 13040-13045.

Uddin, L. Q., Supekar, K., and Menon, V. (2010). Typical and atypical development of functional human brain networks: insights from resting-state fMRI. Front. Syst. Neurosci. 4:21. doi:10.3389/fnsys.2010.00021.

Wang, J.,Zuo, X., and He,Y.(2010). Graphbased network analysis of resting-state functional MRI. Front. Syst. Neurosci. 4:16. doi:10.3389/fnsys.2010.00016.

Conflict of Interest Statement: The authors declare that the research was conducted in the absence of any commercial or financial relationships that could be construed as a potential conflict of interest.

Received: 27 June 2010; accepted: 05 July 2010; published online: 11 August 2010. Citation: Uddin LQ and Menon V (2010) Introduction to special topic-resting-state brain activity: implications for systems neuroscience. Front. Syst. Neurosci. 4:37. doi: 10.3389/fnsys.2010.00037

Copyright (c) 2010 Uddin and Menon. This is an open-access article subject to an exclusive license agreement between the authors and the Frontiers Research Foundation, which permits unrestricted use, distribution, and reproduction in any medium, provided the original authors and source are credited. 